\title{
Assessment of atrial septal defect using 2D or real-time 3D transesophageal echocardiography and outcomes following transcatheter closure
}

\author{
Bingqing Deng ${ }^{1,2 *}$, Kequan Chen ${ }^{3 \#}$, Tucheng Huang ${ }^{1,2 *}$, Yulin Wei ${ }^{1,2}$, Yingmei Liu ${ }^{1,2}$, Li Yang $^{1,2}$, \\ Qiong Qiu ${ }^{1,2}$, Shaoxin Zheng ${ }^{1,2}$, Hanlu $\mathrm{Lv}^{1,2}$, Peiwei Wang ${ }^{1,2}$, Ruqiong Nie ${ }^{1,2 *}$, Jingfeng Wang ${ }^{1,2 *}$ \\ ${ }^{1}$ Cardiovascular Department, Sun Yat-sen Memorial Hospital, Sun Yat-sen University, Guangzhou, China; ${ }^{2}$ Guangzhou Key Laboratory of Molecular \\ Mechanism and Translation in Major Cardiovascular Disease, Sun Yat-sen Memorial Hospital, Sun Yat-sen University, Guangzhou, China; \\ ${ }^{3}$ Department of Gastroenterology, the First Affiliated Hospital of Guangzhou Medical University, Guangzhou, China \\ Contributions: (I) Conception and design: B Deng; (II) Administrative support: J Wang, R Nie; (III) Provision of study materials or patients: T Huang; \\ (IV) Collection and assembly of data: B Deng, Y Wei, Y Liu, L Yang, Q Qiu, S Zheng, H Lv, P Wang; (V) Data analysis and interpretation: B Deng, \\ K Chen; (VI) Manuscript writing: All authors; (VII) Final approval of manuscript: All authors. \\ \#These authors contributed equally to this work. \\ *These authors contributed equally to this work as corresponding authors. \\ Correspondence to: Ruqiong Nie; Jingfeng Wang. Cardiovascular Department, Sun Yat-sen Memorial Hospital, Sun Yat-sen University, Guangzhou \\ 510120, China. Email: nieruqiong@163.com; wjingf@mail.sysu.edu.cn.
}

Background: The assessment of interatrial septum (IAS) requires a standardized, systematic approach, including two-dimensional transthoracic echocardiography (2D TTE), 2D transesophageal echocardiography (2D TEE), and three-dimensional (3D) TEE. Although 2D TEE has been widely used for the preoperative assessment of atrial septal defect (ASD), its ability to provide reliable information is often limited due to the structural characteristics of IAS. The introduction of 3D TEE provides a unique "en face" view of IAS, which allows the visualization and accurate measurements of diameters, area, and rims of ASD. Hence, appropriate ASD imaging information is particularly important in successful transcatheter closure.

Methods: In this retrospective study, 2D TTE/TEE, and 3D TEE were performed before ASD closure, with 2D minimal and maximal diameters, areas, and residual rims being recorded. Adequate $3 \mathrm{D}$ TEE imaging data sets were collected and then analyzed. ASD related parameters were compared using different echocardiography. Patients who underwent ASD closure completed a clinical follow-up.

Results: The mean defect maximal diameter and aperture area by $3 \mathrm{D}$ TEE was significantly larger than that of the corresponding 2D TEE $(\mathrm{P}<0.05)$. There was no statistical difference in the minimal and maximal diameter or area by TEE for circular-shaped ASDs. For oval ASDs, mean minimal diameter on 2D TEE was larger than that on 3D TEE. The mean maximal diameter measured using 2D TEE was smaller than the $3 \mathrm{D}$ TEE measurement $(16.0 \pm 7.1$ vs. $19.8 \pm 8.6$; $\mathrm{P}<0.05)$. For complex-shaped defects, there were statistical differences in minimal and maximal diameter between TEEs. Furthermore, 2D and 3D TEE had a longer superior vena cava (SVC) residual rim than did 2D TTE $(\mathrm{P}<0.05)$. The 3D TEE residual rims of the inferior vena cava (IVC) was significantly larger than the corresponding 2D TEE. There was a very strong correlation between the residual rim measurements using 3D and 2D TEE. However, the limits of agreement between 2D and real-time 3D TEE measurements were more apparent in the IVC rim group than in the other groups.

Conclusions: Our study confirms the value of 3D TEE in assessing ASD shape and size reported by previous studies, and is also the first to accurately and systematically characterize ASD residual rim in complex ASDs.

Keywords: Atrial septal defect (ASD); real-time three-dimensional transesophageal echocardiography (real-time 3D TEE); two-dimensional transesophageal echocardiography (2D TEE); transcatheter closure 
Submitted Jun 02, 2021. Accepted for publication Aug 05, 2021.

doi: $10.21037 / \mathrm{atm}-21-3206$

View this article at: https://dx.doi.org/10.21037/atm-21-3206

\section{Introduction}

Atrial septal defects (ASDs) are one of the most common congenital heart defects and account for approximately $6-10 \%$ of congenital heart disease cases (1). The assessment of atrial septum abnormalities and associated complications requires a systematic and standardized approach, including the use of two-dimensional (2D) transthoracic echocardiography (TTE), 2D transesophageal echocardiography (TEE), and real-time three-dimensional (3D) TEE (2).

The interatrial septum (IAS) is a very dynamic and complex 3D anatomic structure. Although 2D TEE has widely been used for the assessment of ASD, it is often unable to provide reliable information on the minimal and maximal diameters and the size of the area of defect (3). Moreover, 2D echocardiography has limitations in evaluating the IAS, as it does not exist in a flat plane that can be easily aligned or interrogated by 2D echocardiography. The recent introduction of real-time 3D matrix array TEE transducers could provide unique views from the left atrial or right atrial perspective of the IAS. This "en face" makes the visualization of ASDs possible, while the multiplanar reconstruction (MPR) modality also allows for the measurement of ASD diameters, area, and rims. Furthermore, real-time 3D TEE can display the dynamic nature of the atrial defect size during cardiac cycles.

Transcatheter closure of ASD has become a popular approach in surgical repair with favorable clinical outcomes. Studies have confirmed the clinical significance of $3 \mathrm{D}$ TEE for the guidance of surgical occlusion of ASDs. The comprehensive ASD imaging information provided by 3D TEE, including location, shape, size, area, and the neighboring residual rim conditions is imperative for successful transcatheter closure. In the absence of proper preoperative evaluation, the wrong choice of device for transcatheter device may lead to major complications. This occurs when the chosen device is oversized or too small. An oversized device could increase the risk of device erosion, especially in defects with aortic rim deficiency (4). Conversely, a small size occluder could lead to residual shunts or device embolization, and could even require emergency surgical operation. Therefore, the determination of the right occlusion device size is crucial for transcatheter closure.

The appropriate ASD device selection is based on realtime 3D TEE and 2D balloon stretched diameter (SBD) measurements. SBD measuring and balloon sizing are complicated and time-consuming processes, and thus exploring the reliability of 3D TEE imaging for selecting the ASD device size is highly desirable. This involves investigating the relationship between $3 \mathrm{D}$ TEE and balloon size, and identifying those defect signs (5) that correlate with the balloon size.

Several studies have found a relationship between the ASD diameter, size, and area assessed by 3D TEE, and the SBD measured with the balloon sizing method. However, only regular-shaped ASDs, including circular and oval ASDs, were included in these studies. The relationships between parameters measured by 2D TEE and real-time 3D TEE in complex ASDs, including those with irregular shapes and special positions, still need to be explored, especially the rims in the complex secundum ASDs. Thus, this study assessed the maximal and minimal diameters, the area, and the residual rims by $2 \mathrm{D}$ and $3 \mathrm{D}$ TEE, and investigated the relationship between these measurements in patients with complex secundum ASDs. We also evaluated the outcomes of patients, including TTE immediately and 1 day after ASD closure, and at least 1 TTE examination between 1 and 3 months following device closure.

Our study aimed to (I) compare standard 2D TEE methods and real-time 3D TEE in measuring minimal and maximal aperture diameters and area in ASD patients, and (II) to quantify the ability of real-time 3D TEE to measure rim length in complex ASD while clarifying residual shunting outcomes after the transcatheter closure. We present the following article in accordance with the STROBE reporting checklist (available at https://dx.doi. org/10.21037/atm-21-3206).

\section{Methods}

\section{Study population}

For the study population, 81 patients who presented with secundum ASDs between August 2018 to February 2021 were enrolled in our retrospective study at the Department of Cardiology, at Sun Yat-sen Memorial 

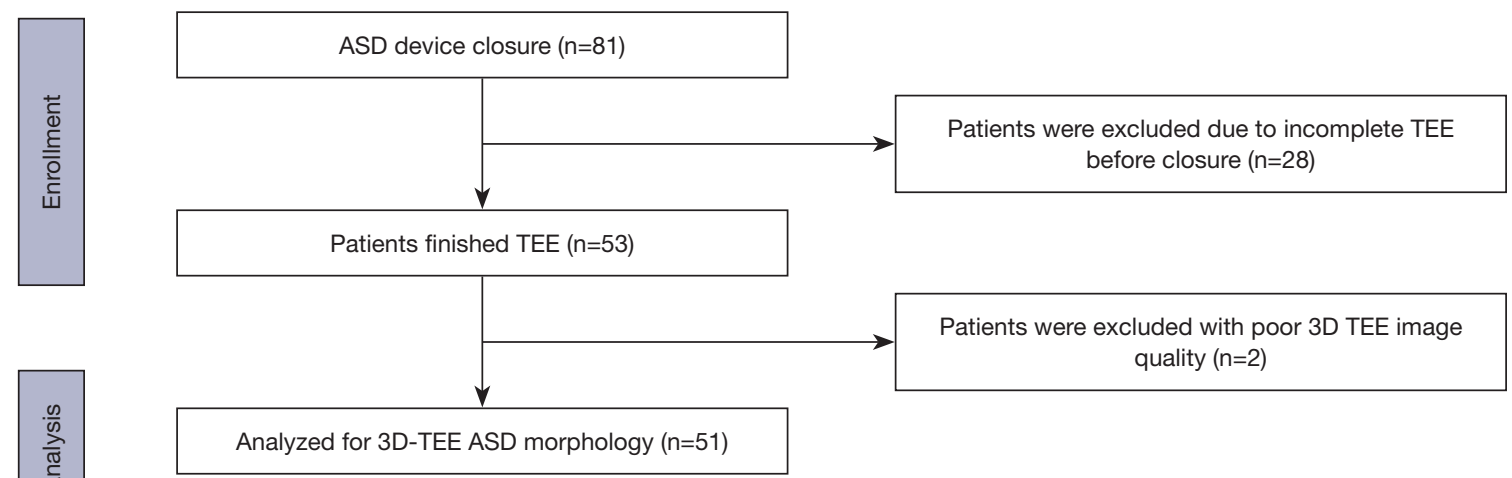

Figure 1 Research flowchart: overview of the enrollment process and analysis of this study cohort. In the study, 81 patients underwent ASD closure. A total of 51 2D TTE/2D TEE/RT-3D TEE imaging data sets were found to be of acceptable quality. ASD, atrial septal defect; 2D, two-dimensional; TTE, transthoracic echocardiography; RT, real-time; 3D, three-dimensional; TEE, transesophageal echocardiography.

Hospital, Sun Yat-sen University. All participants underwent ASD closure with a septal occluder (Lifetech Company, Shenzhen, China). However, 28 patients were excluded due to incomplete TEE before closure. The remaining patients underwent comprehensive multiplane $2 \mathrm{D}$ and $3 \mathrm{D}$ TEE procedures, and two of these patients were excluded due to inadequate $3 \mathrm{D}$ TEE image quality. 3D TEE was included in the TEE procedure throughout the recorded data sets, which were analyzed offline; hence, the acquisition of 3D imaging did not extend TEE time significantly. Criteria for the evaluation of transcatheter ASD closure included a minimal rim of $5 \mathrm{~mm}$ of septal tissue around the defect to allow for occlusion device stability. The device was also adequately separated from other important adjacent structures. Figure 1 shows the enrollment flowchart of this cohort. This study complied with the Declaration of Helsinki (as revised in 2013), and the study was approved by the ethics committee of Sun Yat-sen Memorial Hospital, Sun Yat-sen University (No. SYSEC-KY-KS-2021-131). Informed written consent for TEE was obtained by all patients.

\section{Echocardiography protocols}

Standard 2D TTE examinations were performed using Philips Elite echocardiograph with X5-1 probe (Philips Healthcare, Washington, USA) and Siemens SC2000 echocardiograph with 4V1C probe (Siemens Medical Systems, Erlangern, Germany). TEE procedures were performed while the patients were under local infiltration oropharyngeal anesthesia with lidocaine mucilage.
Patients fasted for 12 hours before the TEE procedure. The vital signs, including heart rate, blood pressure, electrocardiogram, and finger vein blood oxygen saturation were closely monitored during the operation. TEE imaging was conducted using Philips Elite echocardiography with X7-2t live 3D TEE transducer and Siemens SC2000 echocardiography system with a Z6Ms live 3D TEE transducer. All the TEE imaging was acquired according to the American Society of Echocardiography (ASE) standard protocol $(6,7)$. ASD diameters and shapes were visually recognized and measured by $2 \mathrm{D}$ TEE with $0-180^{\circ}$ angles, including a four-chamber view $\left(0^{\circ}\right)$, a short-axis view $\left(30-60^{\circ}\right)$, and a long-axis view $\left(90-120^{\circ}\right)$. Continuous rotation of 2D TEE facilitated the measurement of the minimal or maximal ASD diameters. 2D Maximal and minimal and diameters were recorded respectively at the cardiac end-systolic frame cycle when the defect sizes appeared largest (Figure 2A,2B). The 2D ASD area was calculated from the ellipse formula by measuring width and length (8). The 3D TEE full volume modality was then switched, or a real-time zoom 3D box including the whole ASD was positioned (Figure 2C,2D). Finally, adequate 3D TEE imaging data sets were collected. The 3D gain and compression were optimized to guarantee the image quality. $3 \mathrm{D}$ volume rotations allowed for left-right (Figure 2D) or right-left en face views of the ASD, providing for a quick and visual analysis of its shape and its adjacent surrounding structures (Figure 2E-2H). A horizontal plane parallel to the IAS plane allowed for the display of the ASD en face from the right or left atrium. Simultaneously, two planes orthogonal to the horizontal axis of the atrial septum, 

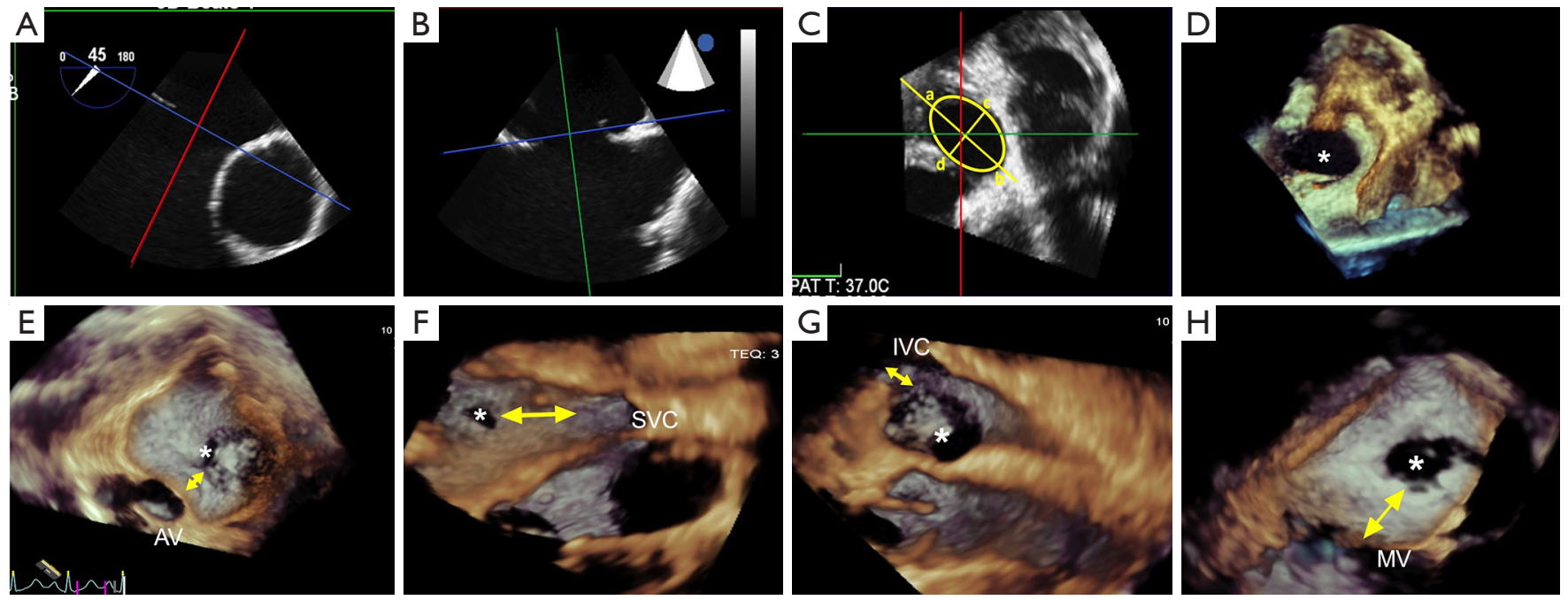

Figure 2 Representative measurements of ASD minimal and maximal diameters, and area, using MPR and the zoom 3D TEE volume modality. (A) The plane cropping through the aperture in the anterior-posterior dimension. (B) The plane cropping through the defect in the inferior-to-superior dimension. (C) A case where the minimal diameter was overestimated and the long-axis diameter was underestimated by 2D TEE. The green line (supposedly short axis) and the red line (supposedly long axis) depict how the corresponding 2D TEE images are unable to illustrate the real minimal and maximum diameter of this defect. (C) The multiplanar reconstructed views of an elliptical ASD. Lines $\mathrm{a}-\mathrm{b}$ and $\mathrm{c}-\mathrm{d}$ represent the real long and short axis of this elliptical-shaped ASD. The yellow circle represents the real outline of this defect. (D) Reconstructed 3D TEE view of an ASD seen en face from the left atrium. If an en face plane is selected using multiple planes as described in (C), the defect's area is calculated by planimetry. The maximal (length) and minimal (short) axis can also be measured as shown. The yellow arrow head in (E-H) illustrate the residual rim of the aorta, SVC, IVC, and MV annulus, respectively. A white asterisk represents ASD. ASD, atrial septal defect; MPR, multiplanar reconstruction; 3D, three-dimensional; TEE, transesophageal echocardiography; 2D, two-dimensional; SVC, superior vena cava; IVC, inferior vena cava; MV, mitral valve; AV, aortic valve.

known also as the bicaval view, were used to display the en face plane which was parallel to the ASD. TEE operators could easily describe ASD morphology as oval, round, or complex based on these en face views. The data sets were stored digitally, transferred to an echo workstation, and then analyzed by offline software. TEE operations were completed by three senior operators with similar experience, and catheterizations were performed by an experienced interventional cardiologist.

\section{$2 D$ and 3D TEE data analyses}

For 2D ASD imaging, both minimal and maximal ASD aperture diameters were measured at the end of the cardiac systole frame (9), as the shape and size of the aperture dynamically changes throughout the cardiac cycle $(10,11)$. Measurements of the defect area and residual tissue rims from the en face view were also acquired during the largest defect aperture at the end of cardiac systole. We defined end-systole as the frame showing the onset of the aortic valve closing motion in the $3 \mathrm{D}$ imaging data set. From either 3D zoom or full volume data sets, the maximum diameter (length) in the long axis, minimal diameter (width) in the short axis, and aperture area by planimetry were measured from the $3 \mathrm{D}$ en face view (Figure 2D) with offline QLAB software version 7.0 (Philips Medical Systems). En face views of the aperture were reconstructed from at least 5 cardiac cycles. ASD morphology was further categorized by the shape seen from the en face view. We defined a circular ASD as a circular or near circular defect (Figure 3A) when the minimal diameter was equal or greater than $75 \%$ of the maximum diameter. An elliptical- or oval-shaped defect was defined as a defect with a minimal length smaller than $75 \%$ of the maximum diameter (Figure 3B) (12). For the purposes of this study, we defined complex ASDs by their shape and/or position. Irregular, asymmetric-shaped defects which were not elliptical or round were defined as complex-shaped ASD. Complex ASDs also included double- or multihole ASD, atrial septum aneurysm with multi perforations, and 

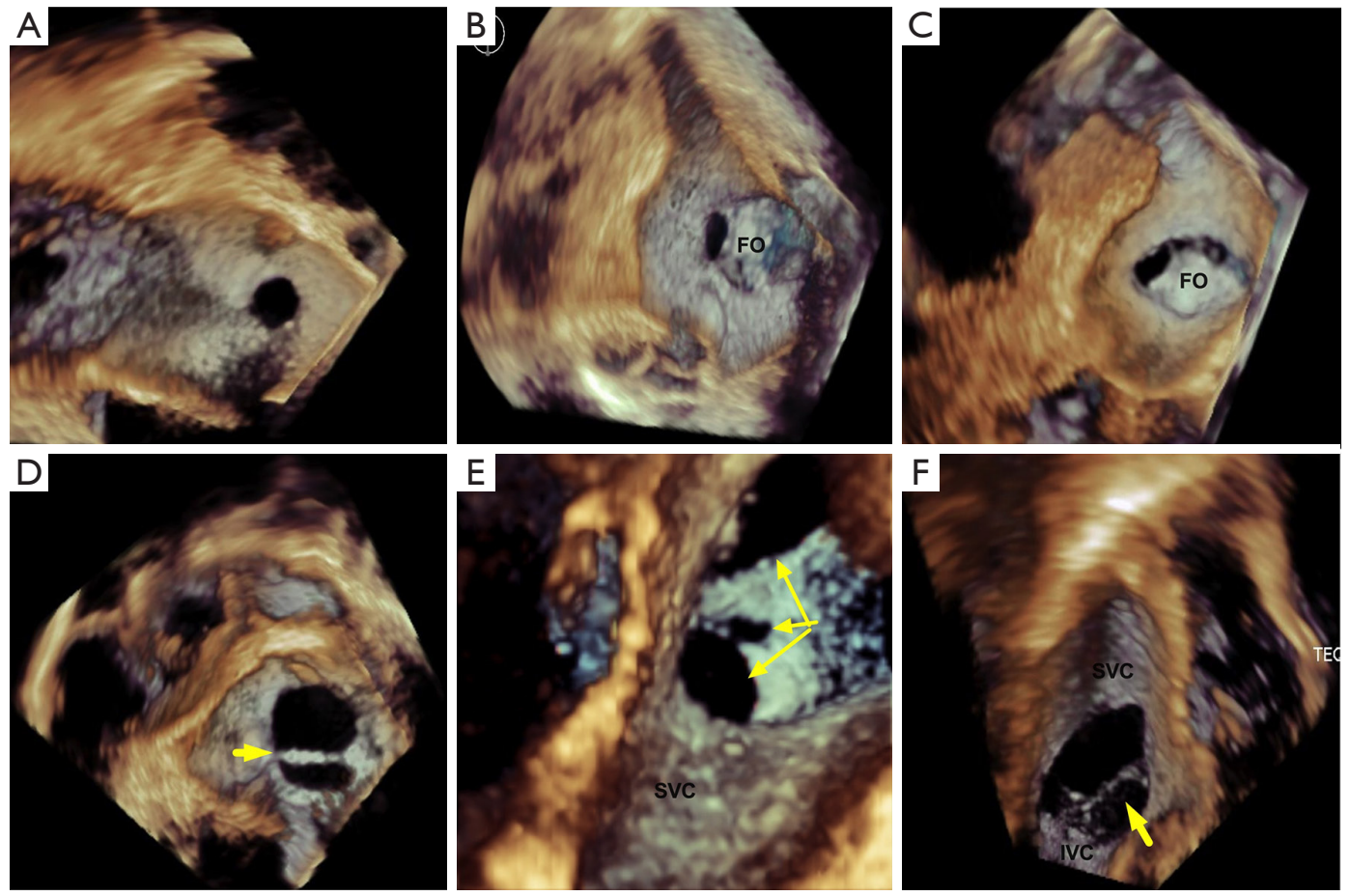

Figure 3 Representative figures of different types of ASD. (A) An ASD with a nearly circular shape. (B) A small ASD with an elliptical shape. (C) An irregular ASD with a crescent shape. (D) An ASD with double-large orifices; the yellow arrow head points to the residual atrial tissue.

(E) Atrial septum aneurysm with multiple perforations; the yellow arrow heads point to the defects: 2 are large; 1 is small. (F) An ASD with a floppy residual rim tissue which is close to the IVC [yellow arrow in (F)] and with a firm residual rim on the side of the SVC. ASD, atrial septal defect; SVC, superior vena cava; IVC, inferior vena cava; FO, foramen ovale.

defects close to the superior vena cava (SVC) or inferior vena cava (IVC). Examples of these complex situations are shown in Figure 3C-3F. The ASD area was calculated using $2 \mathrm{D}$ imaging data sets with the ellipse formula being $0.785 \times$ minimum diameter (width) $\times$ maximum diameter (length). The real-time 3D TEE ASD area was traced at the end of systole frame throughout two cardiac cycle.

\section{Definition of residual rims}

The ASD residual rims were defined as the distance between the ASD edges and the surrounding anatomic tissues. To measure the ASD residual rims, 3D full volume data sets were sliced or cropped. The maximal rim length of the aorta, SVC, IVC, and mitral valve annulus were measured at end cardiac systole (Figure 2E-2H). We measured the postero-superior or postero-inferior (13) residual rim as distance from the defect edge to the SVC or IVC by cable view at the mid-high esophageal position, antero-superior aorta residual tissue rim as the ASD edge to the aorta wall by aortic short axis view at highesophageal position, and antero-inferior (13) residual rim as distance from the ASD rim to the mitral valve annulus by four-chamber cardiac view at the middle position of the esophagus.

\section{Transcatheter ASD closure}

Transcatheter ASD device closure was performed under TEE guidance and fluoroscopy which have already been proved safety and efficacy. Occlusion device size selection was dependent on $2 \mathrm{D}$ and $3 \mathrm{D}$ TEE echocardiographic measures, regardless of whether or not stretched balloon sizing was used. The appropriate occlusion device should be large enough to cover the entire defect and engage the defect's residual rims but small enough to avoid squeezing of adjacent structures and increasing the erosion risk. 2D TTE imaging was collected 1 day after closure, before discharge, and 3 months after closure. Data were analyzed for any evidence of residual shunting. 
Table 1 Clinical characteristics of 51 patients for ASD device closure in this study

\begin{tabular}{lc}
\hline Clinical parameter & Value \\
\hline Age $(\mathrm{y})$ & $46.0 \pm 18.9[15-85]$ \\
Men & $13(25.5)$ \\
Women & $38(74.5)$ \\
Atrial fibrillation & $8(15.7)$ \\
Diabetes mellitus & $5(9.8)$ \\
Hypertension & $7(13.7)$ \\
Primary indication for ASD closure & \\
TIA/stroke & $6(11.8)$ \\
Tricuspid valve regurgitation and PAH & $25(49.1)$ \\
Symptoms with right heart dilation & $32(62.7)$ \\
Symptoms without right heart dilation & $19(37.3)$ \\
\hline
\end{tabular}

Data are expressed as numbers (\%) or mean \pm SD [range]. Symptoms included decreased exercise tolerance, dyspnea on exertion, and syncope. ASD, atrial septal defect; TIA, transient ischemic attack; PAH, pulmonary hypertension.

\section{Statistical analysis}

All statistical analyses were performed using GraphPad version 9.0 (GraphPad, San Diego, CA, USA). Continuous quantitative variables are expressed as mean \pm standard deviation (SD). Categorical variables are presented as counts and percentages. Comparisons between measurements were performed using $t$-tests and linear regression analysis. The Shapiro-Wilk test was used to test the normality of the distribution of the variables. Levene's test was used to assess the homogeneity of variance. If these conditions were not satisfied, a nonparametric Wilcoxon test was used for the bivariate analysis. The mean values in the subgroups were compared using one-way analysis of variance and post hoc analyses. Pearson correlation was used to assess the correlations between variables. Bland-Altman analysis was used to evaluate the agreement between two different measurement parameters. A $\mathrm{P}$ value $<0.05$ was considered statistically significant.

\section{Results}

\section{Study population}

Our retrospective study included a total of 51 patients who accepted TEE examination and ASD closure. Clinical baseline characteristics are shown in detail in Table 1. Among the patients, 38 patients were female $(74.5 \%)$ and $13(25.5 \%)$ were male. The mean age was $46.0 \pm 18.9$ [15-85] years. Atrial fibrillation, diabetes mellitus, and hypertension were present in $8(15.7 \%), 5(9.8 \%)$, and $7(13.7 \%)$ patients, respectively. The primary indications for ASD closure were right ventricular chamber dilatation, with symptoms including decreased exercise tolerance, dyspnea on exertion, and syncope in $32(62.7 \%)$ patients; pulmonary hypertension (PAH) in 25 (49.1\%) patients; absence of right ventricular chamber dilation in 19 patients $(37.3 \%)$; and stroke or transient ischemic attack (TIA) from paradoxical embolism in 6 patients $(11.8 \%)$ (Table 1$)$.

\section{ASD shape and size}

Of all the ASD patients, only $8(15.7 \%)$ had circular-shaped defects (Figure 3A), 12 (23.5\%) had oval-shaped defects (Figure $3 B$ ), and $31(60.8 \%)$ had defects that were complex in shape or position (Figure $3 C-3 F$ ). Representations of these complex ASDs are shown in Figure 3C-3F, including asymmetry, irregularly shaped defects, double holes, atrial septum aneurysm with multiple perforations, and defects very close to the aorta, SVC, or IVC.

\section{ASD diameter and area}

The mean defect maximal diameter (length) measured using real-time 3D TEE was significantly larger than that of corresponding 2D TEE $(\mathrm{P}<0.05)$. However, there was no difference in mean ASD minimal diameter (width) between 2D TEE imaging or corresponding real-time 3D TEE imaging. The measurements of mean aperture area were also significantly different between these two modalities: $2.2 \pm 1.5 \mathrm{~cm}^{2}$ as calculated by planimetry from left-right en face view using $3 \mathrm{D}$ TEE, and $1.6 \pm 1.2 \mathrm{~cm}^{2}$ as calculated by the ellipse formula with 2D TEE (Table 2). When ASD shape and position factors were considered, the results differed greatly. Compared with 2D TEE, there was no statistical difference in the minimal and maximal diameter or area by real-time 3D TEE for circular-shaped ASDs. For oval-shaped ASDs, the mean minimal diameter measured by $2 \mathrm{D}$ TEE was significantly larger than that of the corresponding 3D TEE $(13.9 \pm 7.2 v s .9 .1 \pm 6.4 \mathrm{~mm}$; $\mathrm{P}<0.05)$. Similarly, the mean maximal diameter measured by $2 \mathrm{D}$ TEE was significantly smaller than that of $3 \mathrm{D}$ TEE $(16.0 \pm 7.1$ vs. $19.8 \pm 8.6 \mathrm{~mm} ; \mathrm{P}<0.05)$. However, there was no difference either by $2 \mathrm{D}$ or real-time $3 \mathrm{D}$ TEE in oval- 
shaped aperture area $\left(2.0 \pm 1.7\right.$ vs. $\left.2.1 \pm 1.4 \mathrm{~cm}^{2} ; \mathrm{P}>0.05\right)$. For complex-shaped defects, 2D TEE and real-time 3D TEE showed statistically significant differences in minimal and maximal diameter, and area. Both the maximal and minimal diameter were larger when measured with real-time 3D TEE (maximal diameter: $22.4 \pm 7.7$ vs. $17.3 \pm 8.8 \mathrm{~mm}$;

Table 2 Average minimal and maximal dimension, and area of ASD measured by $2 \mathrm{D}$ TEE and $3 \mathrm{D}$ TEE $(\mathrm{n}=51)$

\begin{tabular}{lcc}
\hline Defects size & 2D TEE & 3D TEE \\
\hline Minimal dimension $(\mathrm{mm})$ & $12.1 \pm 6.0$ & $10.2 \pm 3.8$ \\
Maximal dimension $(\mathrm{mm})$ & $16.2 \pm 6.6$ & $20.2 \pm 7.3^{\star}$ \\
Area $\left(\mathrm{cm}^{2}\right)$ & $1.6 \pm 1.2$ & $2.2 \pm 1.5^{\star}$ \\
\hline
\end{tabular}

All data are presented as mean \pm SD. The 2D TEE defect area was obtained from an ellipse area calculation. 3D TEE defect en face area was acquired from direct 3D planimetry. ${ }^{*}, P<0.05,3 D$ TEE measurement significantly larger than the corresponding 2D TEE. ASD, atrial septal defect; 2D, two-dimensional; TEE, transesophageal echocardiography; 3D, three-dimensional; SD, standard deviation. minimal diameter: $15.7 \pm 5.9$ vs. $12.0 \pm 6.5 \mathrm{~mm} ; \mathrm{P}<0.05)$ and similarly, the aperture area measured by $2 \mathrm{D}$ TEE was smaller than that measured by $3 \mathrm{D}$ TEE $(\mathrm{P}<0.05$; Table 3$)$.

\section{ASD residual rims}

To determine the effect of the defect location, we specifically investigated residual rims of the complex ASDs. There were 31 complex ASD patients in all. We measured ASD margin, including IVC, SVC, mitral valve annulus, and aortic rims. Table 4 shows the mean value of residual rims measured using 2D TTE, 2D TEE, and real-time 3D TEE. According to the location of ASD, 20 defects were near the aorta and had a short residual rim of less than $5 \mathrm{~mm}$, 6 defects were close to the SVC, and 5 were close to the IVC. There were no statistically significant differences in aorta or mitral valve annulus residual rim measurements between the 3 echocardiographic methods $(\mathrm{P}=0.29$ and $\mathrm{P}=0.33$, respectively). In regard to the SVC residual rim, 2D TTE had a shorter rim than did 2D and 3D TEE, with the latter having an average SVC residual rim of $10.4 \pm 5.6$

Table 3 Average minimal and maximal dimension, and area for circular, oval, and complex ASD by 2D TEE and 3D TEE

\begin{tabular}{lccc}
\hline Defects size & Circular $(\mathrm{n}=8)$ & Oval $(\mathrm{n}=12)$ & Complex $(\mathrm{n}=31)$ \\
\hline 2D minimal dimension $(\mathrm{mm})$ & $9.1 \pm 3.3$ & $13.9 \pm 7.2^{\dagger}$ & $12.0 \pm 6.5$ \\
3D minimal dimension $(\mathrm{mm})$ & $8.9 \pm 4.5$ & $9.1 \pm 6.4$ & $15.7 \pm 5.9^{\star}$ \\
2D maximal dimension $(\mathrm{mm})$ & $11.7 \pm 3.9$ & $16.0 \pm 7.1$ & $17.3 \pm 8.8$ \\
3D maximal dimension $(\mathrm{mm})$ & $12.9 \pm 5.1$ & $19.8 \pm 8.6^{\star}$ & $22.4 \pm 7.7^{\star}$ \\
2D area $\left(\mathrm{cm}^{2}\right)$ & $1.2 \pm 0.5$ & $2.0 \pm 1.7$ & $1.7 \pm 1.2$ \\
3D area $\left(\mathrm{cm}^{2}\right)$ & $1.3 \pm 0.7$ & $2.1 \pm 1.4$ & $2.8 \pm 1.4^{*}$ \\
\hline
\end{tabular}

All data are presented as mean $\pm \mathrm{SD} .{ }^{\dagger}, \mathrm{P}<0.05,2 \mathrm{D}$ TEE measurement significantly larger than corresponding 3D TEE; ${ }^{\text {, }} \mathrm{P}<0.05,3 \mathrm{D}$ TEE measurement significantly larger than the corresponding 2D TEE. ASD, atrial septal defect; 2D, two-dimensional; TEE, transesophageal echocardiography; 3D, three-dimensional; SD, standard deviation.

Table 4 Rims of complex ASD measured by 2D TTE, 2D TEE, and 3D TEE

\begin{tabular}{|c|c|c|c|c|}
\hline Defects rims & 2D TTE & 2D TEE & 3D TEE & $\mathrm{P}$ \\
\hline SVC residual rim (mm) & $7.9 \pm 6.0$ & $10.4 \pm 5.6^{\star}$ & $11.9 \pm 4.7^{\star}$ & $<0.05$ \\
\hline Mitral valve annulus rim (mm) & $4.9 \pm 3.3$ & $5.2 \pm 3.7$ & $5.3 \pm 3.0$ & 0.33 \\
\hline Aortic rim (mm) & $3.8 \pm 2.9$ & $4.0 \pm 3.2$ & $4.2 \pm 3.9$ & 0.29 \\
\hline
\end{tabular}

All data are presented as mean $\pm \mathrm{SD}$. * $\mathrm{P}<0.05,2 \mathrm{D}$ TEE significantly larger than the corresponding $2 \mathrm{D}$ TTE measurement; * $\mathrm{P}<0.05,3 \mathrm{D}$ TEE measurement significantly larger than the corresponding 2D TEE measurement. ASD, atrial septal defect; 2D, two-dimensional; TTE, transthoracic echocardiography; TEE, transesophageal echocardiography; 3D, three-dimensional; IVC, inferior vena cava; SVC, superior vena cava; SD, standard deviation. 
and $11.9 \pm 4.7 \mathrm{~mm}$, respectively $(\mathrm{P}<0.05)$. However, there was no difference between both TEE modalities. For the IVC residual rim, both $2 \mathrm{D}$ and $3 \mathrm{D}$ TEE also had a longer rim compared with 2D TTE. The average IVC rims were $9.8 \pm 6.2$ and $12.8 \pm 7.0 \mathrm{~mm}$ for $2 \mathrm{D}$ TEE and $3 \mathrm{D}$ TEE, respectively $(\mathrm{P}<0.05)$. Moreover, the $3 \mathrm{D}$ TEE measurements in the IVC residual rim were significantly larger than the corresponding 2D TEE measurements (Table 4).

\section{Comparison of ASD rim between 2D TEE and real-time 3D TEE}

There was a strong correlation between aortic residual rim tissue measurements with real-time $3 \mathrm{D}$ TEE and 2D TEE, with no statistically significant differences being found between these modalities $\left(r=0.94 ; R^{2}=0.89\right.$; $\mathrm{P}<0.01)$. Similarly, there was a good correlation between these two measurements for the residual rims of SVC and IVC ASDs (SVC rim: $r=0.92, \mathrm{R}^{2}=0.85$; IVC rim: $\mathrm{r}=0.90$; $\left.\mathrm{R}^{2}=0.83 ; \mathrm{P}<0.001\right)$. However, Bland-Altman analysis showed that the limits of agreement between 2D and 3D TEE measurements were broader in the IVC rim group than in the aorta and SVC rim group (Figure 4): aortic and SVC rim measurements showed a mean difference of $-0.21 \mathrm{~cm}$ with $95 \%$ agreement of $\pm 0.51 \mathrm{~cm}$ and $-0.67 \mathrm{~cm}$ with $95 \%$ agreement of $\pm 1.11 \mathrm{~cm}$ (Figure 4 ), respectively; and IVC residual rim measurements showed a mean difference of $-0.32 \mathrm{~cm}$ with a $95 \%$ agreement of $\pm 1.24 \mathrm{~cm}$.

\section{Clinical follow-up}

We examined the 81 ASD occlusion patients who were enrolled in the study from August 2018 to February 2021: 28 patients did not undergo TEE, while 51 patients did. All the patients who underwent ASD closure completed the clinical follow-up, which included 2D TTE immediately and 1 day after ASD closure, as well as before discharge, and 3 months after device closure. Ten patients showed evidence of residual shunting from left atrial to right atrial using TTE assessment immediately after occlusion (10/81, $12.3 \%)$, and eight patients showed mild residual shunting 3 months after the occlusion $(8 / 81,9.9 \%)$. More residual leaks were observed in the patients who did not undergo $3 \mathrm{D}$ TEE examination. We observed that in these 8 patients with residual shunting, 6 did not complete the $3 \mathrm{D}$ TEE exam while the remaining 2 were complex ASD type. Of these 2 complex ASDs, 1 patient had a defect which was very near to the IVC and the other patient had a complex atrial septum aneurysm with multiple perforations.

\section{Discussion}

Compared with cardiac surgery, the transcatheter closure of secundum ASD, which has a lower complication morbidity rate, has already become the preferred mode of repair when the anatomy of atrium permits it $(14,15)$. The atrial septum should be assessed fully with TEE before this invasive procedure is selected. It is always difficult to depict the exact minimal or maximal diameter solely by 2D TEE due to the defect's 3D morphology and its fluctuation up and down, and back and forth during the cardiac cycle. The IAS is also extremely thin, making it more difficult to evaluate the defect. Real-time 3D TEE technology provides vivid imaging that can dynamically display the temporal and spatial changes in defect shape, size, and of surrounding tissue, and clarify the residual rim $(10,16)$.

Commercially available quantitative software is available for measurements of defect diameters and area in 2D imaging extracted from 3D TEE data sets. Moreover, the real-time en face views of the ASD defect can be visualized with high definition from both the left atrial and right atrial perspectives. Defect parameters can also be acquired directly using the $3 \mathrm{D}$ zoom modality. The advantages of real-time $3 \mathrm{D}$ TEE are crucial for comprehending the morphology and position of ASD, especially for complex defects. In addition to this, we find that ASD defect size and shape change dynamically with cardiac cycle changes. Defect size may reach a maximal area at the end of cardiac systole and a minimal area at the end cardiac diastole, which is consistent with previous studies.

Before 3D echocardiography was widely used, operators measured ASD by continuously rotating the multiple plane TEE transducer to find the minimal and maximal planes. The maximal and minimal diameters measured by 3D TEE imaging shows moderate correlations with those measured by $2 \mathrm{D}$ TEE imaging modality, suggesting that the defect diameters measured using 3D TEE modality are comparable to those from 2D TEE measurements. However, in our study a single measurement of ASD diameters using 2D TEE from either a long or short axis view was insufficient to indicate the true maximal or minimal diameter. We found that relying only on 2D TEE produced a larger average minimal defect diameter than did real-time $3 \mathrm{D}$ TEE measurement. Likewise, the average maximal defect diameter measured by 2D TEE was smaller than that measured by real-time 3D TEE, which is consistent with 

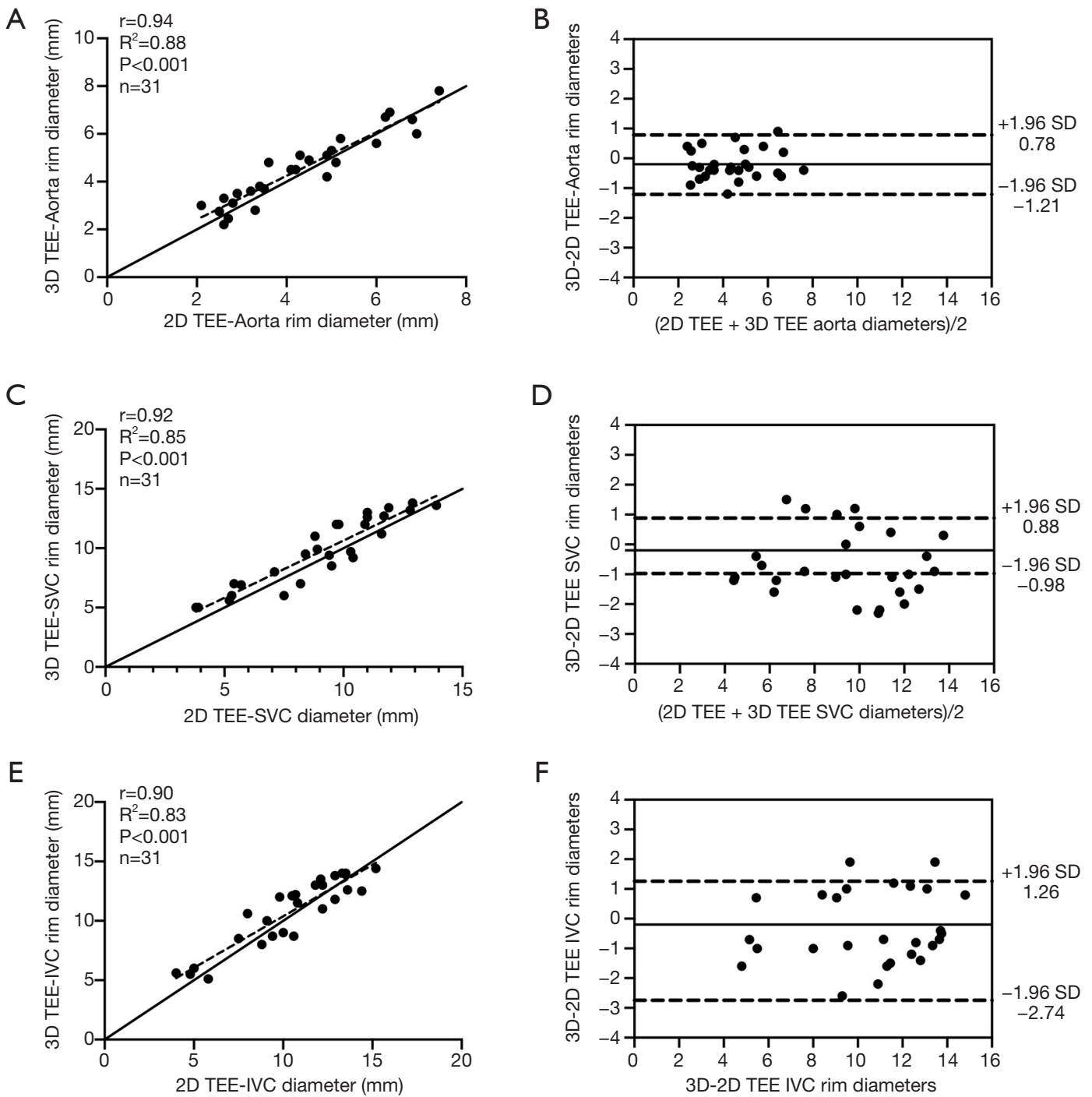

Figure 4 Relationship of the defect residual rim length between 2D TEE and real-time 3D TEE in complex ASD. (A,C,E) Simple linear regression analysis of 2D TEE and real-time 3D TEE measurements in aortic, SVC and IVC rims. (B,D,F) The Bland-Altman analysis of the differences in measurement of residual rims between 2D TEE and real-time 3D TEE. On the correlation graphs (A,C,E), dashed lines represent linear regression while solid lines represent lines of identity. On Bland-Altman plot graphs (B,D,F), solid lines represent average differences, while dashed lines represent average difference $\pm 1.96 \times \mathrm{SD}$ of difference. $2 \mathrm{D}$, two-dimensional; TEE, transesophageal echocardiography; 3D, three-dimensional; ASD, atrial septal defect; SD, standard deviation; SVC, superior vena cava; IVC, inferior vena cava.

previous studies (8). Therefore, 3D TEE must be heavily relied upon to determine the accurate ASD size and choose the proper occlusion size. Moreover, 3D TEE can provide an entire en face/surgical view of an ASD and can easily find the plane with minimal or maximal diameters. However, Maeno et al. (17) reported 3D TEE measurements were smaller than those of 2D TEE, and they stated that the realtime 3D TEE, which measures from the inner margins of the defect rims, would underestimate the maximal diameter.
Acar (18) and Seo (19) et al. reported that the defect difference between $2 \mathrm{D}$ and real-time $3 \mathrm{D}$ measurements was affected by the shape and size of the ASD. They found that the correlation and agreement between the measurements of the two modalities was stronger in a circular-shape group than in an elliptical shape group. In our study, we further classified 51 patients into three groups according to the shape and position: circular-shaped defects, ovalshaped defects, and complex-shaped defects (including 
special position ASDs and irregular-shaped ASDs). Consistent with previous research (20), we found that the agreement between the minimal and maximal diameters, and area measurements under 2D and real-time 3DTEE was stronger in patients with circular-shaped ASDs than in those with elliptical or complex ASDs. For the patients with elliptical-shaped ASDs, we also found the minimal diameter tended to be larger under 2D TEE than under real-time 3D TEE. Conversely, 2D TEE produced a larger maximal diameter. One possible reason for this is that despite continuously rotating the $2 \mathrm{D}$ multiple plane TEE transducer to find the minimal and maximal diameters, it is easy to deviate from the real minimal/maximal plane in the irregular-shaped defects. For the complex ASDs, due to the special position and/or complicated shape, the diameters and area discrepancies are much larger between the different TEE modalities. Therefore, the difference in measurements between these two modalities appears to be impacted by both the position and shape of the defect.

In standard 2D TEE, the cross-orthogonal sectional planes often do not often cut the exact minimal or maximal distance from the defect to the surrounding rim tissues; thus, the rim lengths may not be accurate. This modality should not be solely relied upon, as 2D TEE may misestimate the true defect rim length. 3D TEE full-volume data sets which encompass both the ASD defect and surrounding residual rim structures avoid this misestimation. Given this advantage, cropping 3D pyramidal data sets could easily provide the true length of the defect's residual edges compared with the traditional 2D TEE modality.

The aortic residual rim could be observed clearly with the 2D TTE, 2D TEE, and 3D TEE modalities. This is due to its close acoustic field location. In contrast, it is usually difficult to show the surrounding structure of SVC with 2D TTE in both the inferior-xiphoid double atrial view and the short axis view due to its distal acoustic field. However, these could be perfectly displayed in the cable view using $2 \mathrm{D}$ and $3 \mathrm{D}$ TEE. Due to its residual rim, it is the hardest to show IVC because its position in the acoustic field is the most distant. However, with the help of real-time 3D TEE technology and the intelligent 3D crop software, we could present IVC residual rim much more clearer than ever before. These allow for a more confident selection of occlusion size, especially in patients with an ASD close to the IVC.

In addition, we studied the relationship between $2 \mathrm{D}$ and 3D TEE measurements of the ASD residual rim, especially in complex ASDs. A good linear relationship was also found between these two modalities in measurement of residual rims, which is consistent with previous studies (10). Moreover, we found the limits of IVC rim agreement between 2D and 3D TEE measurements were broader than those of both the aortic and SVC rim groups. This large discrepancy is closely related to the IVC location at the far acoustic field. These findings emphasize the advantages of real-time $3 \mathrm{D}$ TEE in displaying and measuring the residual rim, especially that of the remote IVC, as compared to 2D TEE.

Good quality 3D imaging acquisition requires operators' expertise, and analyzing 3D data sets requires additional time to process. When the quality of the real-time 3D TEE imaging is unsatisfactory, it is important to first guarantee the imaging quality of $2 \mathrm{D}$ TEE. The probe can be gently moved to avoid gas interference. Attention should also be paid to adjusting imaging data sets, which involves intelligent image cropping and echo-gain improvement. It is also critical to prevent insufficient gain, which can result in an extremely floppy atrial septum and can even lead to pseudo-echo loss, which may cause ASD misdiagnosis and an oversized occluder.

In addition to the previously mentioned advantages, there are, of course, disadvantages of 3D TEE, namely the expansive 3D TEE data set collection and tedious offline imaging reconstruction processing. Therefore, detailed $3 \mathrm{D}$ analysis would be better performed in advance of transcatheter closure rather than during the manipulation. Owing to the recent development of matrix array $3 \mathrm{D}$ TEE transducers, the time spent on 3D data set collection and tedious offline imaging reconstruction processing will be greatly shortened. Moreover, with the application of the "D'art" function, defects can be immediately measured with this new cropping software and may provide the detailed occluder selection information to interventional cardiologists. This means that 3D TEE measurements may be relied upon to instantaneously choose the implanted ASD device size during the process of transcatheter closure. This will make this method more clinically acceptable and likely alter future clinical practice.

Moreover, in our research, more than $40 \%$ of the study patients had an antero-superior aorta residual rim length shorter than $5 \mathrm{~mm}$, which is very common in ASDs. This type of ASD is usually very close to the aortic noncoronary sinus. However, it is still feasible for operators to achieve successful occlusion if prudently chosen ASD device size.

The atrial septum is considered to be floppy when the 
septum is extremely thin and when the edges of the defect are mobile. Real-time 3D TEE can easily determine if the defect edge is floppy or firm, which helps operators rationally adjust the occluder size. If the ASD shape is regularly round, with a firm defect, $2 \mathrm{~mm}$ can be added to the chosen occlusion size. In case of a floppy septum, more caution is needed, and we may add up to $4 \mathrm{~mm}$ or more to the occlusion size, depending on the floppy area (21). However, in our center, this practice still has to be validated in future research.

We found that among the 8 postoperative residual shunts patients, up to $70 \%(6 / 8)$ did not accept the $3 \mathrm{D}$ TEE examination before ASD occlusion for a variety of reasons. Further studies are needed to assess whether occlusion device selection depending on 3D TEE would lead to a decrease in residual shunting after transcatheter ASD closure. Besides the size and shape of ASD, other factors may also contribute to the presence of residual shunting, including insufficiency or deficiency of residual rims, the ASD location, and even the device selection method. On the basis of 3D TEE, choosing a larger device size could have resulted in less residual shunting. After occlusion, a tiny amount of residual drainage will be disappear over time. We suspect this may be related to the growth of endothelial cells on the occluder. Moreover, we recognize that our study was retrospective in design and lacked long-term followup data, and thus more cases should be included in future studies.

\section{Conclusions}

With the development of transcatheter treatment for structural heart disease, TEE is playing an increasingly important role in ASD device selection and closure procedure. Simple 2D measurement of ASDs is a quick and reliable method for appropriate occluder size selection, especially if the atrial septum is firm. However, if the configuration of the ASD is more complex (e.g., presence of irregular shape, floppy or multifenestrated IAS positioned near the IVC), 3D echocardiography can offer a visual and detail-rich assessment of the ASD. Our findings are in line with previous results in confirming the value of $3 \mathrm{D}$ TEE on ASD shape and size assessment. A novelty of our study is the accurate and systematic characterization of ASD residual rim in complex ASDs. The residual rims in different directions showed moderate correlation between the 2D and 3D TEE modalities. The limit of agreement is different between 2D and 3D TEE for residues in different directions due to the acoustic field. This methodology provides a more comprehensive understanding and assessment of ASD configuration, which can lead to better occluder device selection and fewer residual shunts for transcatheter closure.

\section{Study limitations}

Our study is limited by its retrospective nature and the small number of enrolled patients. Another limitation is the lack of the validation from repair surgery as the gold standard, as not all of the patients underwent transcatheter intervention. Meanwhile, patients did not undergo preoperative cardiac computed tomography or magnetic resonance imaging, the use of which could have provided detailed information about the shape, size, and residual rims of ASD. Moreover, the major limitation of 3D TEE is the reduced temporal resolution compared to $2 \mathrm{D}$ TEE which means we should assess ASDs with different types of echocardiography. Finally, we did not separate the complex ASDs by position and shape. In future studies, we will consider collecting more cases and conducting the subgroup analysis.

\section{Acknowledgments}

Funding: This work was supported by the National Natural Science Foundation of China (NSFC) grant (No. 81700261). We also acknowledge the grant support from the Guangzhou Science and Technology Plan Project (Livelihood Science and Technology; No. 201903010009).

\section{Footnote}

Reporting Checklist: The authors have completed the STROBE reporting checklist. Available at https://dx.doi. org/10.21037/atm-21-3206

Data Sharing Statement: Available at https://dx.doi. org/10.21037/atm-21-3206

Conflicts of Interest: All authors have completed the ICMJE uniform disclosure form (available at https://dx.doi. org/10.21037/atm-21-3206). The authors have no conflicts of interest to declare.

Etbical Statement: The authors are accountable for all aspects of the work in ensuring that questions related to the accuracy or integrity of any part of the work are 
appropriately investigated and resolved. This study complied with the Declaration of Helsinki (as revised in 2013), and the study was approved by the ethics committee of Sun Yat-sen Memorial Hospital, Sun Yat-sen University (No. SYSEC-KY-KS-2021-131). Informed written consent for TEE was obtained by all patients.

Open Access Statement: This is an Open Access article distributed in accordance with the Creative Commons Attribution-NonCommercial-NoDerivs 4.0 International License (CC BY-NC-ND 4.0), which permits the noncommercial replication and distribution of the article with the strict proviso that no changes or edits are made and the original work is properly cited (including links to both the formal publication through the relevant DOI and the license). See: https://creativecommons.org/licenses/by-nc$\mathrm{nd} / 4.0 /$.

\section{References}

1. Roberson DA, Cui W, Patel D, et al. Three-dimensional transesophageal echocardiography of atrial septal defect: a qualitative and quantitative anatomic study. J Am Soc Echocardiogr 2011;24:600-10.

2. Johri AM, Rojas CA, El-Sherief A, et al. Imaging of atrial septal defects: echocardiography and CT correlation. Heart 2011;97:1441-53.

3. Song BG, Park SW, Lee SC, et al. Real-time 3D TEE for multiperforated interatrial septum. JACC Cardiovasc Imaging 2010;3:1199.

4. Amin Z, Hijazi ZM, Bass JL, et al. Erosion of Amplatzer septal occluder device after closure of secundum atrial septal defects: review of registry of complications and recommendations to minimize future risk. Catheter Cardiovasc Interv 2004;63:496-502.

5. Aggeli C, Apostolou I, Dimitroglou Y, et al. The role of 'halo sign' for the accurate quantification of atrial septal defect size with 3D TEE. Int J Cardiovasc Imaging 2020;36:873-81.

6. Silvestry FE, Cohen MS, Armsby LB, et al. Guidelines for the echocardiographic assessment of atrial septal defect and patent foramen ovale: from the American Society of Echocardiography and Society for Cardiac Angiography and Interventions. J Am Soc Echocardiogr 2015;28:910-58.

7. Saric M, Perk G, Purgess JR, et al. Imaging atrial septal defects by real-time three-dimensional transesophageal echocardiography: step-by-step approach. J Am Soc
Echocardiogr 2010;23:1128-35.

8. Johri AM, Witzke C, Solis J, et al. Real-time threedimensional transesophageal echocardiography in patients with secundum atrial septal defects: outcomes following transcatheter closure. J Am Soc Echocardiogr 2011;24:431-7.

9. Handke M, Schäfer DM, Müller G, et al. Dynamic changes of atrial septal defect area: new insights by threedimensional volume-rendered echocardiography with high temporal resolution. Eur J Echocardiogr 2001;2:46-51.

10. Kaku K, Takeuchi M, Sugeng L, et al. Assessment of atrial septal defect size and residual rim using real-time 3D transesophageal echocardiography. J Echocardiogr 2009;7:48-54.

11. Franke A, Kühl HP, Rulands D, et al. Quantitative analysis of the morphology of secundum-type atrial septal defects and their dynamic change using transesophageal three-dimensional echocardiography. Circulation 1997;96:II-323-7.

12. Song J, Lee SY, Baek JS, et al. Outcome of transcatheter closure of oval shaped atrial septal defect with amplatzer septal occluder. Yonsei Med J 2013;54:1104-9.

13. van den Bosch AE, Ten Harkel DJ, McGhie JS, et al. Characterization of atrial septal defect assessed by real-time 3-dimensional echocardiography. J Am Soc Echocardiogr 2006;19:815-21.

14. Du ZD, Hijazi ZM, Kleinman CS, et al. Comparison between transcatheter and surgical closure of secundum atrial septal defect in children and adults: results of a multicenter nonrandomized trial. J Am Coll Cardiol 2002;39:1836-44.

15. Butera G, Biondi-Zoccai G, Sangiorgi G, et al. Percutaneous versus surgical closure of secundum atrial septal defects: a systematic review and meta-analysis of currently available clinical evidence. EuroIntervention 2011;7:377-85.

16. Roushdy A, El Sayegh A, Ali YA, et al. A novel threedimensional echocardiographic method for device size selection in patients undergoing ASD trans-catheter closure. Egypt Heart J 2019;72:1.

17. Maeno YV, Benson LN, McLaughlin PR, et al. Dynamic morphology of the secundum atrial septal defect evaluated by three dimensional transoesophageal echocardiography. Heart 2000;83:673-7.

18. Acar P, Saliba $Z$, Bonhoeffer $P$, et al. Influence of atrial septal defect anatomy in patient selection and assessment of closure with the Cardioseal device; a three-dimensional transoesophageal echocardiographic reconstruction. Eur 
Heart J 2000;21:573-81.

19. Seo JS, Song JM, Kim YH, et al. Effect of atrial septal defect shape evaluated using three-dimensional transesophageal echocardiography on size measurements for percutaneous closure. J Am Soc Echocardiogr 2012;25:1031-40.

20. Hascoet S, Hadeed K, Marchal P, et al. The relation between atrial septal defect shape, diameter, and area using three-dimensional transoesophageal echocardiography and balloon sizing during percutaneous closure in children.

Eur Heart J Cardiovasc Imaging 2015;16:747-55.

21. Boon I, Vertongen K, Paelinck BP, et al. How to size ASDs for percutaneous closure. Pediatr Cardiol 2018;39:168-75.

(English Language Editors: G. Stone and J. Gray)
Cite this article as: Deng B, Chen K, Huang T, Wei Y, Liu Y, Yang L, Qiu Q, Zheng S, Lv H, Wang P, Nie R, Wang J. Assessment of atrial septal defect using $2 \mathrm{D}$ or real-time $3 \mathrm{D}$ transesophageal echocardiography and outcomes following transcatheter closure. Ann Transl Med 2021;9(16):1309. doi: 10.21037/atm-21-3206 